\title{
The role of attention in visual and auditory suffix effects
}

\author{
GRAHAM J. HITCH \\ University of Stirling, Stirling, Scotland
}

\begin{abstract}
The auditory suffix effect (SE), in which recall of the terminal items of a sequence is impaired by presenting a redundant item at the end of the sequence, has been attributed to the displacement of information from auditory sensory storage. However, the SE may result entirely from unnecessary processing of the redundant item due to a failure of attentional control. Two studies examined this possibility using visual presentation to minimize the importance of sensory storage as a source of information. Experiment I first demonstrated a visual SE and showed that its magnitude did not vary when background illumination was altered, a factor which affects the duration of sensory storage. Experiment II used auditory as well as visual presentation and tested the hypothesis that training subjects to ignore the suffix would reduce the SE. Training was achieved by interpolating redundant items identical to the suffix within sequences. It abolished the visual SE but left the auditory SE unaffected. The visual SE, therefore, is not solely determined by the physical characteristics of the suffix, and cannot be based on erasure in sensory storage. The auditory data, on the other hand, were consistent with the erasure hypothesis. It was concluded that an SE does not of itself demonstrate the involvement of sensory storage, and, in particular, the visual SE appears to reflect the degree to which the redundant item can be excluded from focal attention.
\end{abstract}

Among the most intriguing aspects of short-term memory are the strong and reliable effects of input modality. Following auditory presentation, short-term serial recall is characterized by a pronounced recency effect, while visual presentation produces virtually no recency at all (Conrad \& Hull, 1968; Corballis, 1966; Routh, 1971). According to Crowder and Morton (1969) and a number of other authors, auditory and visual inputs pass through separate sensory stores before entering a common categorization system. The precategorical acoustic store (PAS) is assumed to have a capacity of only a few items and a decay time of the order of seconds (Crowder, 1969; Crowder \& Morton, 1969), whereas the visual sensory store (iconic memory) has as its main feature a very rapid rate of decay, typically less than half a second (Averbach \& Sperling, 1961; Sperling, 1960). The current contents of both stores are masked or displaced by subsequent inputs in the same modality. Thus, in an auditory STM experiment, information about the last few items survives in PAS for some seconds because it is not displaced by further incoming information. This provides an account of the strong auditory recency effect. The absence of such strong recency with visual presentation is attributed to the much shorter duration of iconic storage (Crowder \& Morton, 1969).

In a series of studies, Crowder and Morton (1969) and

This research was supported by a grant from the British Medical Research Council. Experiment I was carried out at the University of Sussex, and the author wishes to thank Maggie Clarke, Thea Fox, and Danya Mann for help in testing subjects. The author also wishes to thank D. E. Broadbent of the MRC Applied Psychology Unit for making facilities available for conducting Experiment II.

The author is now at the MRC Applied Psy chology Unit. 15 Chaucer Road, Cambridge, England.
Morton, Crowder, and Prussin (1971) have shown that the auditory recency effect can be abolished by presenting an additional redundant item (or suffix) at the end of the list of to-be-remembered items. According to them, the further auditory input provided by the suffix displaces information about the last few items from PAS.

The present paper is concerned with an aspect of the suffix effect (SE) which receives insufficient emphasis in the Crowder-Morton theory, namely, attention. It is evident that the disruption produced by a suffix demonstrates a failure to exclude the unwanted item from attention, even though in most experiments the subject knows precisely both what the item will be and when it will occur. One could suppose, to take an extreme position, that the SE is entirely the result of failing to limit attention to the wanted items and has nothing to do with the properties of PAS. The extra processing associated with the attended suffix item could itself be responsible for the lowered recall of the most recently presented items. On this view the suffix behaves like an additional memory item. It is certainly true that many of the factors which reduce the auditory SE are also variables which would render the suffix easy to ignore if attention was set toward the to-be-remembered items. For instance, it is well known that physical cues provide an efficient basis for attentional selection, whereas content cues do not (Broadbent, 1971): Changing the voice or location of the suffix reduces its effect, whereas changing its semantic content makes little difference (Morton et al., 1971). Though Morton (1970) noted the importance of attention as a determinant of the auditory SE, it is not clear whether he appreciated that failure to reject the 


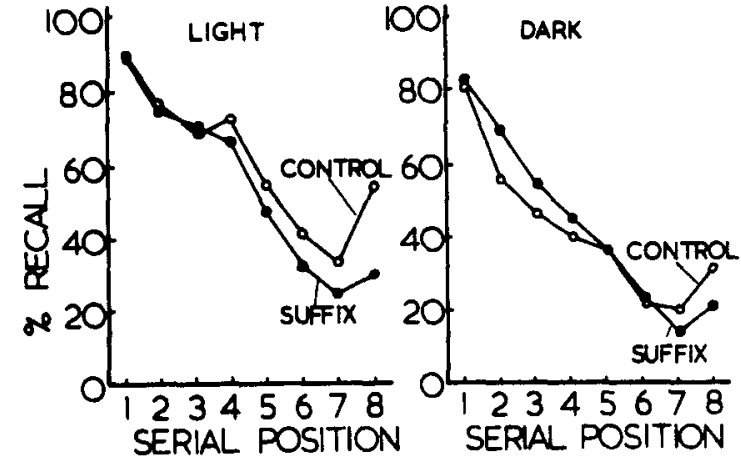

Figure 1. Recall errors as a function of conditions and serial position (Experiment $\mathbf{n}$ ).

suffix at the attentional level might be sufficient for it to occur, with no need to appeal to the properties of PAS.

To test the attentional hypothesis, a visual suffix experiment was carried out. One can arrange for sequential visual presentation of a list of items followed by a suffix under conditions where there is little possibility of the suffix masking the contents of the icon. If there is an SE, it presumably reflects the subjects' failure to ignore the redundant item.

\section{EXPERIMENT I}

Sequences of eight letters were presented visually and sequentially at a rate of two items per second, with or without a suffix. When a suffix did occur, it was presented with the same temporal characteristics as the other items, i.e., a 350-msec exposure followed by a dark ISI of $150 \mathrm{msec}$. Since it was just conceivable that the suffix might have some masking effect with these time intervals, the experiment was run with two levels of background illumination, daylight and total darkness. Item-item masking ought to be greater under the dark viewing conditions, since the ISI was darker and dark postexposure fields maximize the persistence of iconic storage (Sperling, 1960, 1963). An SE based on masking ought, therefore, to be more pronounced in the dark condition.

A second feature of the experiment was that vocal rather than the more usual written recall was required. Thus, in the dark condition the memory items were the subjects' only visual inputs. One might expect the recall of control sequences to show enhanced recency when viewed in the dark, since the icon might be expected to make an appreciable contribution to performance. Surprisingly enough, there is a lack of consistency in the way visual and auditory STM experiments are normally carried out. With auditory presentation, great care is taken to minimize background noise but visual presentation typically occurs in a lighted room providing a large number of irrelevant stimuli. The use of written recall may exacerbate the situation. It is, therefore, just possible that the poor recency normally obtained with visual presentation is only representative of the usual range of testing conditions. The present experiment allows this point to be checked by comparing recall of control sequences under the two viewing conditions.

\section{Method}

Design. The subjects were assigned at random to either the light or dark testing conditions. Each group received six practice trials in which they recalled eight-item letter sequences presented with no suffix. They then received two blocks of 27 sequences, one block containing control sequences and the other containing sequences to which a suffix (the letter "O") was added. The first two sequences of each block served as practice trials. Half of the subjects began with the control and half with the suffix condition. The letter sequences were presented in the same order to all subjects.

Apparatus and materink. The letter sequences were random selections from the set BCFHJQRYZ, subject to the following constraints: (1) No letter was repeated within a sequence and (2) each letter occurred roughly equally often at each serial position. The sequences were coded onto a punched paper tape which programmed a single in-line digital display (Type 650/7, Counting Instruments Ltd.). Each sequence began with a 1.5 -sec warning signal consisting of three successive flahses of a white floodlamp. A tape recorder was used to record the subjects' recall.

Instructions. Subjects were instructed to give verbal recall of the letters in their order of presentation, saying "blank" or guessing whenever they were uncertain of the correct response. Recall began immediately after the offset of the last stimulus item and the next trial began about $8 \mathrm{sec}$ later. On suffix trials, the instructions stressed that the final item would always be the letter " $O$ " and would not have to be recalled. Subjects were asked to cooperate in fixating the visual display and not closing their eyes when the suffix appeared. The experimenter monitored this in the light testing condition but was unable to do so in the dark.

Subjects. The subjects were 24 Sussex University students and were paid for their participation. Twelve were tested in daylight and 12 in darkness.

\section{Results}

The recall data, plotted as serial position curves, are shown in Figure 1. Recall was scored as incorrect for any failure to produce the correct item at the correct position. In both light and dark viewing conditions, the suffix led to a small depression in recall of the final items, the effect being, if anything, slightly greater in the light. The results were subjected to a three-way analysis of variance, with serial position, suffix, and background illumination as factors. The two-way Suffix by Serial Position interaction was highly significant $[F(7,154)=$ $6.4, \mathrm{p}<.001$ ] but the triple interaction (Suffix by Serial Position by Background lllumination) was not $(F<1)$. There were no other significant $F$ ratios apart from the main effects of serial position $[F(7,154)=$ $65.8, \mathrm{p}<.001]$ and background illumination $[\mathrm{F}(1,22)=$ $12.5, \mathrm{p}<.01]$. The latter reflected a generally poorer level of recall under dark viewing conditions, which is difficult to explain other than in post hoc terms.

It is unlikely that visual masking was responsible for the suffix effect (a) because of the temporal intervals used (as argued above) and (b) because there was no sign 
of an increased effect when the experiment was carried out in darkness. The validity of Point $b$ rests on the assumption that subjects in the dark condition were not simply closing their eyes to exclude the suffix on a proportion of trials. Since the experimenter could not monitor for this possibility, a second experiment was carried out to test the masking hypothesis more critically.

The data also show that dark viewing conditions did not give rise to increased recency in the recall of control sequences. This is fortunate, since it suggests that the auditory-visual modality effect in short-term recall is not an artifact of the usual failure to equate the number of irrelevant background stimuli in the two cases. This is also evidence of the unimportance of iconic storage in the task. In darkness, the iconic representation of the final item of control sequences ought to be relatively long lasting, yet this does not benefit its recall.

\section{EXPERIMENT II}

While the first experiment suggests that the visual SE is not connected with sensory storage, it does not demonstrate that the effect is attentional. Experiment II was an attempt to show that a suffix of given physical and temporal characteristics has an effect which changes with manipulations of the subjects' attentional strategy. On a masking hypothesis this ought not to be possible, since masking is generally presumed to be dependent only on physical variables and is not under attentive control. The technique was to examine the result of interpolating a redundant suffix element (the digit zero) before every to-be-remembered item during input. Sequences ended either with or without a zero to give the suffix and control conditions. Using auditory presentation, Hamilton and Hockey (1974) have demonstrated that subjects are able to modulate their attention when critical and noncritical items come in a regular temporal pattern. It therefore seemed probable that with the present visual sequences subjects would adopt a strategy of attending to the wanted items and ignoring the zeros. Thus, a suffix occurring at the end of a sequence should be relatively easy to ignore, and on the attentional hypothesis its effect should be reduced. A set of standard control and suffix sequences did not contain interpolated zeros and were included to provide an estimate of the suffix effect when attention to zeros was not manipulated. The experiment was run using auditory presentation in addition to the visual conditions to see whether the auditory SE behaved in a similar fashion to the visual one.

\section{Method}

Design. Sequences of eight random digits were presented for immediate serial recall in a three-way factorial design. A group testing procedure was used with two main groups $(\mathbb{N}=16$ in each case). One group was presented with sand wich digit strings containing embedded zeros, while the other received standard strings which did not contain interpolated zeros. Each main group was further divided into two equal subgroups to counterbalance the order of presenting the suffix and control conditions and input modality. All groups recalled 72 experimental sequences presented in eight blocks of nine sequences each. There were no repeated digits in any of the sequences, and blocks were arranged so that each digit occurred equally often at each serial position. For half the groups the first four blocks were presented visually and the second four auditorily. For the other half the order was reversed. Each set of four blocks was used to present the suffix and control conditions according to either an SCCS or a CSSC pattern. Half the groups received one pattern and half the other. Though the order of conditions varied from group to group, the actual digit sequences were the same for all groups. They were presented to the different groups in orders which balanced particular sequences across the suffix and interpolated zero variations, but not modality.

Apparatus. As in Experiment I, an in-line visual display was used to present the sequences. For auditory presentation the display was turned away from the subjects and the experimenter read the items aloud as they appeared.

Procedure. Before a new condition was presented, subjects recalled three appropriate practice sequences to familiarize them with the procedure. Digits were presented at a $1 / \mathrm{sec}$ rate in both standard and sandwich sequences. With visual presentation, each digit in a standard sequence was present for $350 \mathrm{msec}$ and was separated from the next digit by a 650 -msec dark ISI. In sandwich sequences the ISI began and ended with 150 -msec dark intervals separated by a 350 -msec presentation of the digit zero. When a suffix was added to the end of a sequence, it occurred after a delay of $150 \mathrm{msec}$ and was presented for $350 \mathrm{msec}$ for both standard and sandwich sequences. The choice of suffix delay was such that the suffix came in rhythm with the previous zeros (when these were present) to maximize the chance of the subjects ignoring it successfully. The waming signal for each visual sequence consisted of three flashes of a white light lasting in all $1.5 \mathrm{sec}$ and about $8 \mathrm{sec}$ were allowed for recall. For auditory presentation, the warning signal consisted of the single word "ready." The temporal intervals were the same as for visual presentation but were subject to a small amount of variability, since the experimenter read the items from the visual display.

Instructions. These were essentially as for the first experiment, with the exception that written rather than spoken recall was required.

Subjects Thirty-two housewives from the subject panel of the Medical Research Council Applied Psychology Unit served as subjects. They were paid for their participation.

\section{Results}

Figure 2 shows the results expressed in terms of the percentage of items correctly recalled at the various serial positions. An error was defined as any failure to produce the correct item at the correct position. Separate analyses of variance were carried out on data collected under the standard and sandwich conditions, respectively.

With standard sequences, both visual and auditory suffix effects were obtained. There was a significant main effect of adding a suffix $[\mathrm{F}(1,15)=35.0$, $\mathrm{p}<.001]$ and a Suffix by Serial Position interaction $[F(7,105)=12.8, p<.001]$. The auditory suffix effect was larger than the visual one $[F(1,15)=31.1$, $\mathrm{p}<.001$ ] but was of a similar form $(F<1$ for the Suffix by Modality by SP interaction). Auditory presentation did not produce superior overall recall 

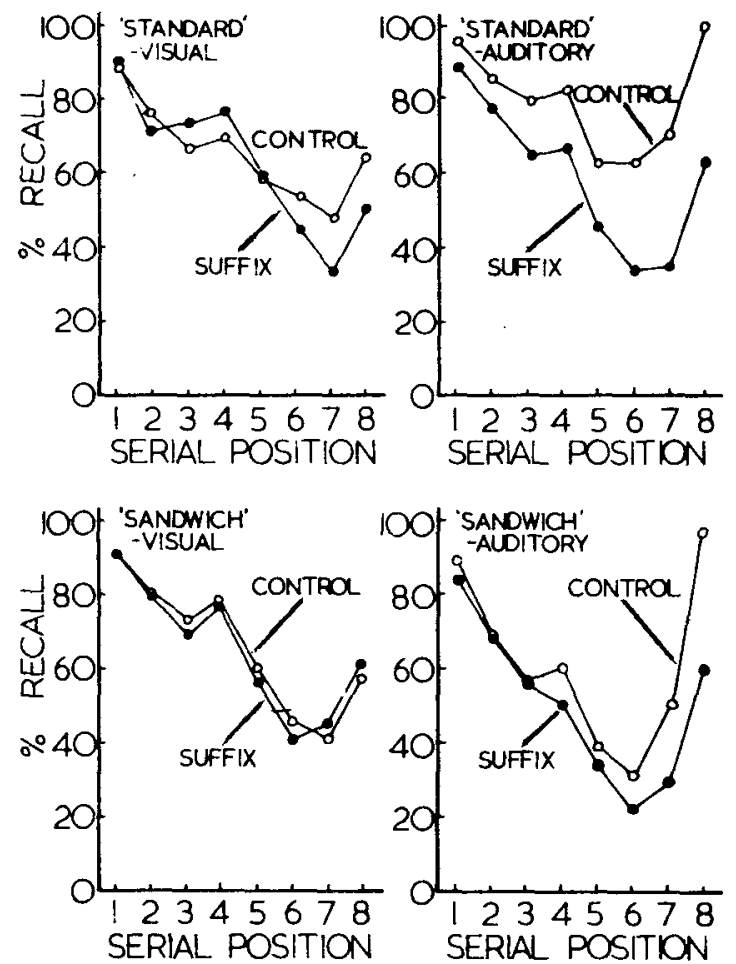

Figure 2. Recall errors as a function of conditions and serial position (Experiment II).

$[F(1,15)=2.0, p>.05]$ but did lead to more recency $[F(7,105)=5.4, p<.001]$. The results obtained with sandwich sequences were quite different. With visual presentation the suffix effect was completely absent, whereas with auditory presentation it was clearly present. This result appeared in the analysis as a three-way interaction between suffix, modality, and SP $[F(7,105)=9.8, p<.001]$. The remaining results of the analysis of variance were similar to those of the previous one, except that auditory presentation led to much poorer recall than visual presentation $[F(1,15)=13.1$, $\mathrm{p}<.01]$. With visual presentation, interpolated zeros had no consequence other than removing the suffix effect, whereas with auditory presentation, recall was lowered at all SPs except the last.

\section{DISCUSSION}

The interpretation of the visual data is quite straightforward. Since the suffix was identical for both standard and sandwich sequences, but its effect was completely absent in the latter, a masking account is untenable. Instead, it is plausible to suppose that the experience of rejecting zeros during the input of sandwich sequences made it easy for the subjects to reject the suffix when it arrived. When standard sequences were presented, the subjects had no such experience, and it was consequently more difficult for them to ignore the suffix. Attentional set is, therefore, a major determinant of the visual SE. This account of the visual data implies that the mere existence of an SE in a modality does not necessarily point to the operation of a sensory store. Thus, it is quite possible that, contrary to their interpretation, Watkins and Watkins' (1974) demonstration of a tactile $\mathrm{SE}$ is not attributable to the retention of raw tactile information.

The attentional hypothesis meets with far less success in accounting for the auditory data. First, the effect of the suffix on recall of the last item presented was independent of whether the sequence was standard or sandwich (see Figure 2). Thus, pretraining subjects to reject zeros did not appear to reduce the size of the SE. Second, sandwich sequences led to poorer recall at all SPs except the last one. On the attentional hypothesis, one could assume that the extra task of ignoring zeros imposed a general overload on the memory system, but this would not account for the absence of increased errors at the last SP. In a quite different test of the attentional account of the auditory SE, Crowder (1971) was also unable to find clear evidence in its favor. On the other hand, both features of the auditory results are broadly consistent with the Crowder-Morton position. Each interpolated zero can be regarded as a suffix for the preceding item, reducing its PAS storage time and, hence, increasing the probability that it is forgotten. A similar effect has been demonstrated by C. Frankish (personal communication), who showed that, with auditory presentation, an isolated within-list suffix lowers recall of the item immediately preceding it. In addition, recall of the final item ought to depend entirely on events following it and not on events prior to it-as the data show. Nevertheless, the attentional hypothesis may have some validity in the auditory case, inasmuch as a failure to reject the suffix would appear to be a prerequisite of it entering PAS.

Since the present experiments were carried out, Neisser and Kahneman (reported in Kahneman, 1973) have confirmed the visual SE using simultaneous rather than sequential presentation. They also take the view that the effect results from a lack of success in excluding the suffix from attentive processing. They showed this by examining the effects of suffixes which differed in the degree to which they were perceptually integrated with the to-be-remembered items. Presumably, a high degree of integration means that the suffix is more difficult to exclude from focal attention. In line with this supposition, the SE was present when the level of integration was high, but appeared to be absent when it was low. These results complement those of Experiment II, where the effect of a given suffix was shown to vary as the subjects' attentional strategy was altered.

In summary, the visual suffix effect appears to be a reliable phenomenon and seems to depend solely on the ease with which the suffix can be rejected at the attentional level. It does not reflect the masking of recently presented items by the redundant item, at least 
at the relatively slow presentation rates used here. At much faster rates, it is highly probable that a masking component would appear. On the other hand, the auditory SE seems to be unlike the visual one in that sensory storage factors are involved. Nevertheless, there are logical grounds for supposing that in this modality, too, attentional failure is an important part of the effect.

\section{REFERENCES}

Averbach, E., \& Sperling, G. Short-term storage of information in vision. In C. Cherry (Ed.), Information theory: Proceedings of the fourth London symposium. London: Butterworth, 1961.

Broadbent, D. E. Decision and stress. London: Academic Press, 1971.

Conrad, R., \& Hull, A. J. Input modality and the serial position curve in short-term memory. Psy chonomic Science, 1968, 10 $135-136$.

Corballis, M. C. Rehearsal and decay in immediate recall of visually and aurally presented items. Canadian Journal of Psy chology, 1966, 20, 43-51.

Crowder, R. G. Improved recall for digits with delayed recal cues. Journal of Experimental Psy chology, 1969, 82, 258-262.
Crowder, R. G. Waiting for the stimulus suffix: Decay, delay, rhythm and readout in immediate memory. Quarterly Joumal of Experimental Psy chology, 1971, 23, 324-340.

Crowder, R. G., \& Morton, J. Precategorical acoustic storage (PAS). Perception \& Psy chophysics, 1969, 5, 365-373.

Hamilton, P., \& Hockey, R. Active selection of items to be remembered: The role of timing. Cognitive Psy chology, 1974, 6, 61-83.

Kahneman, D. Attention and effort. Englewood Clifs, N.J: Prentice-Hall, 1973.

Morton, J. A functional model for memory. In D. A. Norman (Ed.), Models of human memory. New York: Academic Press, 1970 .

Morton, J., Crowder, R. G., \& Prussin, H. A. Experiments with the stimulus suffix effect. Journal of Experimental Psy chology, 1971, 91, 169-190.

Routh, D. A. Independence of the modality effect and amount of silent rehearsal in immediate serial recall. Joumal of Verbal Learning and Verbal Behavior, 1971, 10, 213-218.

Sperling, $G$. The information available in brief visual presentations. Psychological Monographs, 1960, 74, No. 11.

Sperling, G. A model for visual memory tasks. Human Factors, $1963,5,19-31$

Watkins, M. J.. \& Watkins, O. C. A tactile suffix effect. Memory \& Cognition, 1974, 2, 176-180.

(Received for publication September 7, 1974; revision received November 1,1974 .) 